\title{
PREDICTING EXTINCTIONS AS A RESULT OF CLIMATE CHANGE
}

\author{
Mark W. Schwartz, ${ }^{1,5}$ Louis R. Iverson, ${ }^{2,3}$ Anantha M. Prasad, ${ }^{2}$ Stephen N. Matthews, ${ }^{2,3,4}$ \\ AND RAYMOND J. O'CONNOR ${ }^{4}$ \\ ${ }^{1}$ Department of Environmental Science and Policy, University of California, Davis, California 95616 USA \\ ${ }^{2}$ USD A Forest Service, Northeastern Research Station, Delaware, Ohio 43015 USA \\ ${ }^{3}$ School of Natural Resources, Ohio State University, Columbus, Ohio 43210 USA \\ ${ }^{4}$ Department of Wildlife Ecology, University of Maine, Orono, Maine 04469-5755 USA
}

\begin{abstract}
Widespread extinction is a predicted ecological consequence of global warming. Extinction risk under climate change scenarios is a function of distribution breadth. Focusing on trees and birds of the eastern United States, we used joint climate and environment models to examine fit and climate change vulnerability as a function of distribution breadth. We found that extinction vulnerability increases with decreasing distribution size. We also found that model fit decreases with decreasing distribution size, resulting in high prediction uncertainty among narrowly distributed species. High prediction uncertainty creates a conservation dilemma in that excluding these species under-predicts extinction risk and favors mistaken inaction on global warming. By contrast, including narrow endemics results in overpredicting extinction risk and promotes mistaken inaction on behalf of individual species prematurely considered doomed to extinction.
\end{abstract}

Key words: climate and environmental models; climate change; distribution breadth; eastern United States; endemic; extinction; prediction uncertainty; regression tree; vulnerability.

\section{INTRODUCTION}

A striking conclusion that emerges from research predicting effects of climatic warming on biodiversity is that the magnitude of climate-driven extinctions is potentially very large (Schwartz 1992, Berry et al. 2002, Hannah et al. 2002, Midgley et al. 2002, Peterson et al. 2002, Thomas et al. 2004). These estimates are sufficiently alarming to raise international environmental concern (Sala et al. 2000, Millennium Ecosystem Assessment 2005). Although research methods vary, studies that assess the extinction-forcing potential of climatic change assume climatic control of species distributions and use models that include climate to predict vulnerability (Berry et al. 2002, Pearson et al. 2002, Thuiller et al. 2004). The efficacy of using bioclimatic models to assess the possible extinction potential of climate change, particularly among species with small distributions, requires empirical assessment.

We document two fundamental patterns associated with predicting extinction vulnerabilities that, together, decrease our confidence in predicted extinction probabilities for species with narrow distributions. First,

Manuscript received 9 August 2005; revised 9 January 2005; accepted 23 January 2005. Corresponding Editor: D. P. C. Peters.

${ }^{5}$ E-mail: mwschwartz@ucdavis.edu predicted vulnerability to extinction is correlated with range size such that the smaller the geographic extent of a species distribution, the higher the fraction of range displacement as a consequence of warming. Predicted extinction likelihood is higher among species with small current distributions and large range displacement values (Berry et al. 2002, Hannah et al. 2002, Midgley et al. 2002, Peterson et al. 2002, Thomas et al. 2004).

Second, joint climatic and environmental models of the distribution of narrowly distributed species often exhibit poor fit. This is not unexpected, given that many narrowly distributed species are habitat specialists. Species cannot have narrower fundamental niches than their realized niche, but they can have broader ones (Malanson 1997). Narrowly distributed species are probably a mixed suite of species, some of which are limited by climatic tolerances while others are limited by the distribution of edaphic conditions. This latter group, although clearly having some climatic tolerance limits, might not be well predicted by bioclimatic models that assume climatic constraint on current distribution. These observations are of concern because if species that are disproportionately at risk are also those for which we have poor predictive models, then general statements regarding the broad impact of warming on species losses are likely to carry high uncertainty. 


\section{Materials And Methods}

We used data on 142 tree and 116 bird species to assess the relationship between range size and the percentage of the current distribution overlapping the predicted potential future distribution under general climate change models. Distributions and abundance data for trees and birds emerge from the Forest Inventory Analysis and Breeding Bird Surveys, respectively (Iverson et al. 1999, Matthews et al. 2004). These studies provide geographically explicit estimates of current and future tree and bird abundances. These abundance values were summarized on the county level for birds and the subcounty level $\left(400-\mathrm{km}^{2}\right.$ grid cells) for trees. Tree and bird species were included only if the majority of their distribution was located within the United States and east of the 100th meridian, allowing our models to capture information on a large portion of the species distributions. In addition to these criteria, birds that currently have ubiquitous distributions across the eastern United States, along with substantial portions of their distributions expending into Canada and the western United States, were also included. We felt that these species were valid to include in the analysis because they occupy the full extent of our study area and provide the maximum amount of information for generating models.

Joint climate, vegetation, and environment regression tree models were developed for bird species in the eastern United States for the purpose of predicting the distribution of habitat suitability (Breiman et al. 1984, Clark and Pregibon 1992). For tree species, models were developed with 36 climate and environmental variables, using an ensemble regression tree technique called Random Forests (Breiman 2001; Prasad et al. 2006). Both tree and bird models predict the distribution of habitat suitability (Iverson et al. 1999, Matthews et al. 2004). Regression tree procedures recursively separate a response variable, in this case predicted abundance, into increasingly homogenous groups defined by the predictor variables (Breiman et al. 1984, Clark and Pregibon 1992). The resulting model defines terminal groups in terms of a combination of decision rules based on threshold values for the selected predictor variables and, for our application, predicts species abundance for each terminal group as the mean abundance in all cases satisfying that combination of criteria. In Random Forests, bootstrap samples are drawn to construct numerous trees; each tree is grown with a randomized subset of predictors. Fit is then determined by averaging replicate runs. Random Forest results in better prediction accuracy with minimal overfitting than simple classification trees (Breiman 2001; Prasad et al. 2006). Regression tree procedures are one among several classes of species distribution modeling techniques (Guisan and Thuiller 2005). Our intent is not to compare model efficacy, as has been done elsewhere, but simply to demonstrate the logical consequence of small range size on modeling climatic response and to discuss the implications of this observation.

We predicted future potential suitable habitat distributions for tree species by building contemporary models in terms of a suite of five climatic and 25 environmental driver variables. We used seven potential climatic, four elevation, and 68 tree species abundances as driver variables for regression tree modeling of birds. The climatic variables used are also available from climatic models of future conditions. For the bird models, the tree species abundances under future conditions are also available as predictor variables. We then applied the decision rules of the contemporary model onto the predicted future landscapes. This procedure assumes that the contemporary relationships between the response and predictor variables are fixed and that climate change follows the climate change scenarios modeled. We considered doubled $\mathrm{CO}_{2}$ climatic scenarios developed by the UK's Meteorological Office in Hadley (HADCM2SUL; Mitchell et al. 1995) and by the Canadian Climate Center (CGCM1; Boer et al. 2000), these being near opposite ends of the typical spectrum of scenarios used in climate change studies. Again, modeling the full spectrum of climate models is not our primary concern. Rather, we wish to assess the likely consequence of range size on model fit. These climate-habitat models, in general, explain 55-90\% of the variation in current distributions for widely $(>1 \times$ $10^{6} \mathrm{~km}^{2}$ ) distributed species (Iverson et al. 1999, Matthews et al. 2004).

\section{RESUlts}

With the Hadley HADCM2SUL model, both trees and birds showed a significant positive relationship between range size and overlap of current and predicted future ranges $\left(r^{2}=0.46, n=142, P<0.001\right.$ for trees; $r^{2}=$ $0.35, n=116, P<0.001$ for birds; Fig. 1). Predictions of future range overlap with current distributions (for trees, $r^{2}=0.27, P<0.001$; for birds, $r^{2}=0.30, P<$ $0.001)$ were very similar for the CGCM1 model. In each case, species with small range sizes are more likely to have predicted future distributions that are largely or wholly disjunct from current distributions. As a consequence of low overlap between current and predicted distributions, these species are modeled to be exposed to a greater extinction risk as a consequence of dispersal failure or small residual range size.

Second, we examined the relationship between range size and model fit for trees and birds by using joint climate and environment regression tree models (Iverson et al. 1999, Matthews et al. 2004). In particular, we asked whether the proportion of variance in abundance explained systematically increases with increasing distribution breadth (range size). Within a guild of grassland species in the conterminous United States, fit and range size previously have been shown to be positively correlated (Spearman's rho $=0.73, P<0.002$; O'Connor et al. [1999]). For our data sets, both trees and birds 


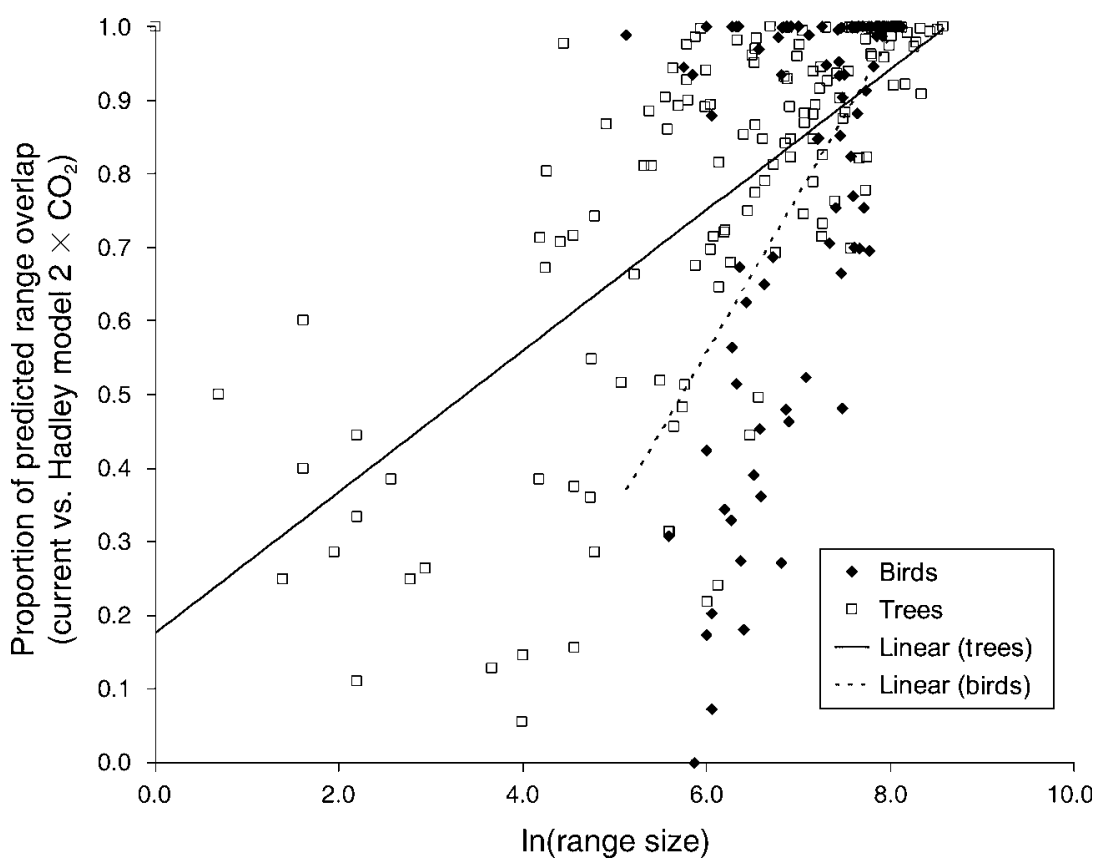

FIG. 1. A scatterplot of the proportion of the current distribution of bird (closed diamonds, $\left.r^{2}=0.39, n=121, P<0.001\right)$ and tree (open squares, $r^{2}=0.40, n=142, P<0.001$ ) species that are overlapped by predicted future distributions (using the Hadley $2 \times$ $\mathrm{CO}_{2}$ model). Range size (log-transformed) was originally measured in thousands of square kilometers. All birds and trees are characterized by having the majority of their current distributions in the eastern United States. Predicted future distributions were estimated using regression tree classification methods.

demonstrate a positive linear relationship between range size and model fit $\left(r^{2}=0.40, n=142, P<0.001\right.$ for trees; $r^{2}=0.31, n=116, P<0.001$ for birds; Fig. 2).

Finally, our data suggest that for species with small distributions, climatic attributes contribute less to overall model fit. For our tree data, climatic variables contribute significantly less to model fit for regression trees of species with smaller range sizes $\left(r^{2}=0.145, n=\right.$ 142, $P<0.001$; Fig. 3).

\section{DisCUSSION}

The general pattern that emerges is that species with small ranges have high predicted climate change vulnerabilities (i.e., low overlap between current and predicted future distributions, but poor model fit and low explanatory value of climatic variables). There are two distinct reasons why model fit may be poor among species with small distributions. Narrowly distributed species, because of few observations, may have low

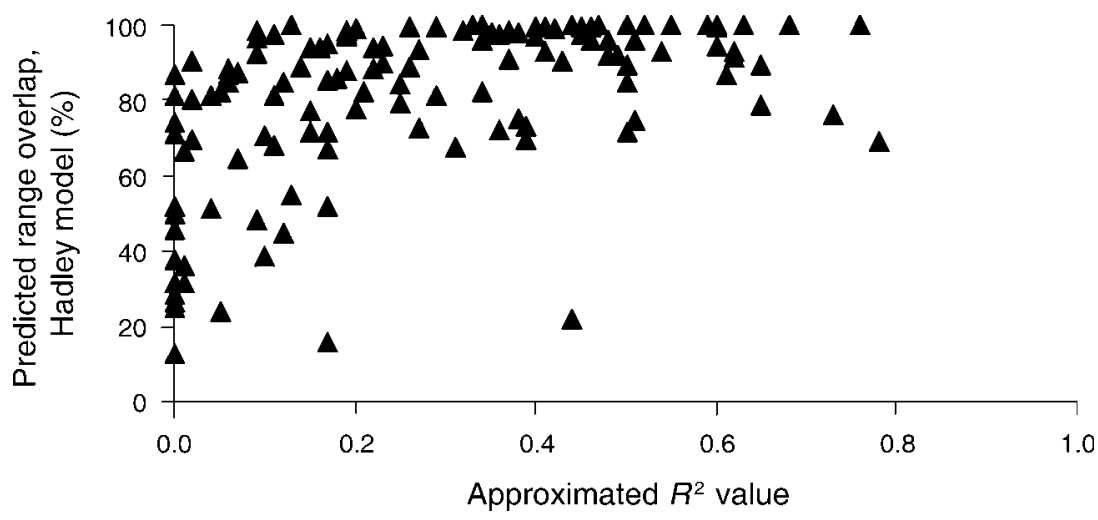

FIG. 2. A scatterplot depicting the decline in predicted range overlap as a function of regression tree model fit for 142 tree species of the eastern United States. Low model fit significantly predicts low overlap between current and predicted future distributions for both the Hadley $\left(r^{2}=0.32, n=142, P<0.001\right)$ and the Canadian Climate Center (Boer et al. [2000], not pictured; $\left.r^{2}=0.25, n=142, P<0.001\right)$ model. 


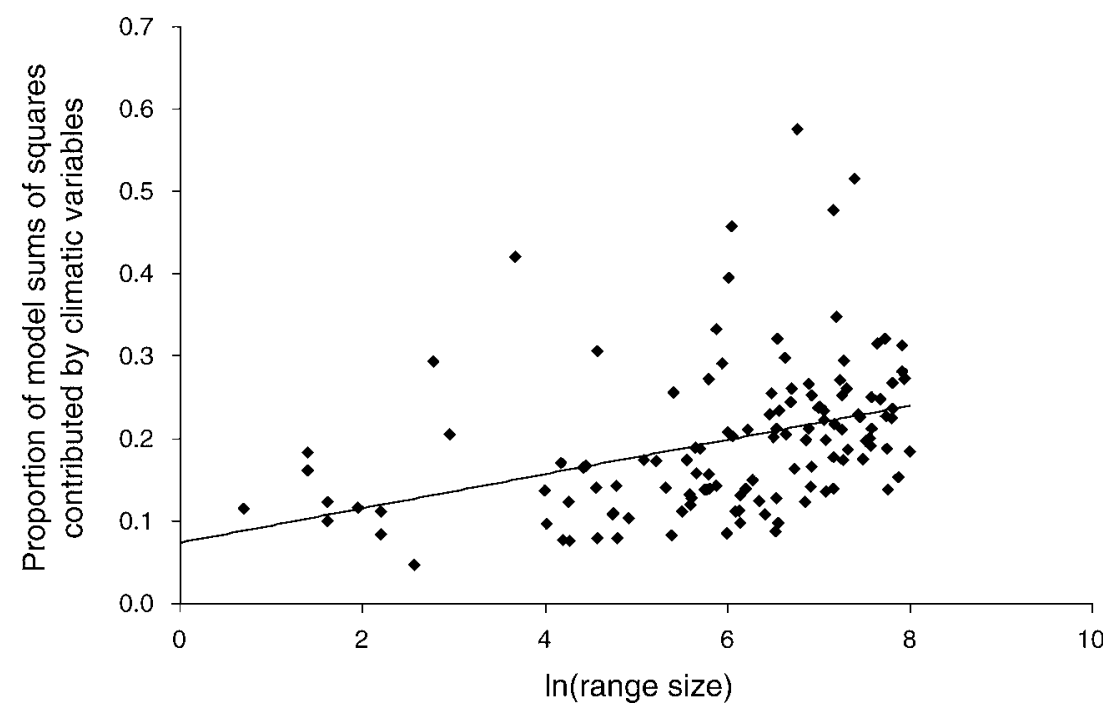

FIG. 3. A scatterplot depicting the smaller contribution of climatic variables to regression tree models predicting the distribution of abundance for tree species with smaller range sizes $\left(r^{2}=0.145, n=142, P<0.001\right)$. Range size (log-transformed) was originally measured in thousands of square kilometers.

statistical power. Nevertheless, Stockwell and Peterson (2002) and others have demonstrated that species distribution models can result in good predictive power even with modest (e.g., 50) sample sizes. A second reason for poor model fit relates to the actual factors limiting species distributions. If a narrowly distributed species is primarily constrained by non-climatic attributes (e.g., soil parent material or disturbance), then climatic attributes might realistically have low explanatory success independent of sample size and statistical power. We argue that this is often the case and must be considered when applying bioclimatic models for conservation application.

To put our observation into perspective, Florida leads the eastern United States with 55 federally listed threatened or endangered plants, of which $87 \%$ are endemic to the state (USFWS 2005). None of the 45 tree species that we consider here with distributions of $<130000 \mathrm{~km}^{2}$ (Florida is $139671 \mathrm{~km}^{2}$ ) had a mean average fit greater than 0.3. Although many Florida endemics have been suggested as being vulnerable to climate change (Box et al. 1999), our results suggest that we may have an insufficient understanding of the climatic responsiveness of this most vulnerable component of biological diversity.

The Monteverde golden toad (Bufo periglenes), a tropical montane endemic, has become the poster child of climate-change-driven extinction (e.g., Bush 2002). Nevertheless, this species may be more of an exception than the rule. We should expect that endemic species may have distributions that are not well predicted by bioclimatic models, because many narrow endemics are limited by non-climatic attributes (e.g., California serpentine endemics). What the actual climatic constraints are on these species is unknown and may depend heavily on responses of potential competitors. For example, many of the endangered plants endemic to the Florida sandhills have demonstrable sensitivity to reductions in fire frequency (Weekley and Menges 2003). Although climate models generally predict increases in fire frequency, fire in this ecosystem is largely under anthropogenic control. In this case, bioclimatic modeling, predicting distributional shifts would, at best, predict extinction vulnerabilities indirectly; the distribution of sand hills and fire is of primary importance.

The Henslow's Sparrow (Ammodramus henslowii) provides another example of the modeling difficulties characteristic of rare species. Although this species is found from Oklahoma to New York, it is distributed in small patches and its actual occupied range is the sixth smallest of the birds modeled here. This patchy distribution resulted in a model that showed poor association with the sparrow's current distribution (model $r^{2}=0.30$ ). When the species' model was projected onto the two climate change scenarios, there was no overlap with its current range. This prediction seems unlikely, given that the species presently occupies such a broad range of climatic conditions. In fact, the real drivers of the species' distribution are linked to grasslands, which were not available as predictors in the model. With declining grassland habitat, this species is vulnerable to extinction (Herkert et al. 2003), but the role of climate change in that vulnerability is uncertain.

Sparse and endemic species are important components of predicting of extinction risk, but they also create problems for predictive bioclimatic modeling. Predictions of extinction threat caused by global climate change are likely to carry high uncertainty because of endemic species. This uncertainty creates a conservation dilemma. Modeling efforts, such as that by Thomas et 
al. (2004) predicting that climate change will drive narrowly distributed species extinct, suggest appropriate action regarding climatic warming, but may foster inappropriate decisions regarding conservation of individual species.

Conservation management has already shifted its emphasis away from narrowly endemic small populations (Schwartz 1999) based, in part, on ecological theory suggesting that these species may be unsustainable. If one asserts that narrowly endemic species are doomed to extinction by climate change, then logic dictates that we either begin programs of assisted migration or divert conservation resources away from these "doomed" species. Divesting of in situ conservation efforts on behalf of narrow endemics as a consequence of warming must be regarded as premature without specific evidence of climatic sensitivity.

Claiming that a particular endemic species is at extinction risk as a consequence of climatic change requires a detailed understanding of the responsiveness to climate of the target species, as well as that of species with which it is likely to interact (Davis et al. 1998). This presents both a daunting task and an opportunity for ecologists; endemic species may provide early warning signals for climate change as an extinction driver because they will be the first to move outside their modeled climatic envelope.

\section{AcKnowledgments}

We thank the USDA Forest Service for support of this research. Sadly, R. O'Connor passed away during the preparation of this manuscript. The other authors express condolences to his family and dedicate this paper to his memory.

\section{Literature Cited}

Berry, P. M., T. R. Dawson, and R. A. Harrison. 2002. Modelling potential impacts of climate change on the bioclimatic envelope of species in Britain and Ireland. Global Ecology and Biogeography 11:453-462.

Boer, G. J., G. M. Flato, and D. Ramsden. 2000. A transient climate change simulation with historical and projected greenhouse gas and aerosol forcing: projected climate for the 21st century. Climate Dynamics 16:427-451.

Box, E. O., D. W. Crumpacker, and E. D. Hardin. 1999. Predicted effects of climate change on distributions of ecologically important native tree and shrub species in Florida. Climatic Change 41:213-248.

Breiman, L. 2001. Random forests. Machine Learning 45:5-32.

Breiman, L., J. H. Friedman, R. A. Olshen, and C. J. W. B. Stone. 1984. Classification and regression trees. Wadsworth, Pacific Grove, California, USA.

Bush, M. B. 2002. Distributional change and conservation on the Andean flank: a paleoecological perspective. Global Ecology and Biogeography 11:463-473.

Clark, L. A., and D. Pregibon. 1992. Tree-based models. Pages 377-419 in T. J. Hastie, editor. Statistical models. Wadsworth, Pacific Grove, California, USA.

Davis, A. J., L. S. Jenkinson, J. H. Lawton, B. Shorrocks, and S. Wood. 1998. Making mistakes when predicting shifts in species range response to global warming. Nature 391:783786.
Guisan, A., and W. Thuiller. 2005. Predicting species distributions: offering more than simple habitat models. Ecology Letters 8:993-1009.

Hannah, L., G. F. Midgley, and D. Millar. 2002. Climate change-induced conservation strategies. Global Ecology and Biogeography 11:485-495.

Herkert, J. R., D. L. Reinking, D. A. Wiedenfeld, M. Winter, J. L. Zimmerman, W. E. Jensen, E. J. Finck, R. R. Koford, D. H. Wolfe, S. K. Sherrod, M. A. Jenkins, J. Faaborg, and S. K. Robinson. 2003. Effects of prairie fragmentation on the nest success of breeding birds in the midcontinental United States. Conservation Biology 17:587-594.

Iverson, L. R., A. M. Prasad, B. J. Hale, and E. K. Sutherland. 1999. Atlas of current and potential future distributions of common trees of the eastern United States. Northeastern Research Station, USDA Forest Service, Radnor, Pennsylvania, USA.

Malanson, G. P. 1997. Simulated responses to hypothetical fundamental niches. Journal of Vegetation Science 8:307316.

Matthews, S. N., R. J. O'Connor, L. R. Iverson, and A. M. Prasad. 2004. Atlas of climate change effects in 150 bird species of the eastern United States. Northeastern Research Station, USDA Forest Service, Radnor, Pennsylvania, USA.

Midgley, G. F., L. Hannah, D. Millar, M. C. Rutherford, and L. W. Powrie. 2002. Assessing the vulnerability of species richness to anthropogenic climate change in a biodiversity hotspot. Global Ecology and Biogeography 11:445-451.

Millennium Ecosystem Assessment. 2005. Ecosystems and human well-being: biodiversity synthesis. World Resources Institute, Washington, D.C., USA.

Mitchell, J. F. B., T. C. Johns, J. M. Gregory, and S. Tett. 1995. Climate response to increasing levels of greenhouse gases and sulphate aerosols. Nature 376:501-504.

O'Connor, R. J., R. B. Boone, M. T. Jones, and T. B. Lauber. 1999. Linking continental climate and land use patterns with grassland bird distribution in the conterminous United States. Studies in Avian Biology 19:45-59.

Pearson, R. G., T. P. Dawson, P. M. Berry, and P. A. Harrison. 2002. SPECIES: A spatial evaluation of climate impact on the envelope of species. Ecological Modelling 154:289-300.

Peterson, A. T., M. A. Ortega-Huerta, J. Bartley, V. SanchezCordero, J. Soberon, R. H. Buddemeier, and D. R. B. Stockwell. 2002. Future projections for Mexican faunas under global climate change scenarios. Nature 416:626-629.

Prasad, A. M., L. R. Iverson, and A. Liaw. 2006. Random forests for modeling the distribution of tree abundances. Ecosystems 9:181-199.

Sala, O. E. et al. 2000. Biodiversity: global biodiversity scenarios for the year 2100. Science 287:1770-1774.

Schwartz, M. W. 1992. Potential effects of global climate change on the biodiversity of plants. Forestry Chronicle 68: 462-471.

Schwartz, M. W. 1999. Choosing an appropriate scale for conservation reserves. Annual Review of Ecology and Systematics 30:83-108.

Thomas, C. D. et al. 2004. Extinction risk from climate change. Nature 427:145-148.

Thuiller, W., M. B. Araujo, R. G. Pearson, R. J. Whittaker, L. Brotons, and S. Lavorel. 2004. Biodiversity conservation: uncertainty in predictions of extinction risk. Nature 430: 6995.

USFWS (U.S. Fish and Wildlife Service). 2005. Endangered species website. 〈http://endangered.fws.gov/wildlife. html\#Species $\rangle$

Weekley, C. W., and E. S. Menges. 2003. Species and vegetation responses to prescribed fire in a long-unburned, endemic-rich Lake Wales Ridge scrub. Journal of the Torrey Botanical Society 130:265-282. 